\title{
A Narrative Study on Postmodern War Fiction Si je t'oublie, Bagdad
}

\author{
Ying Huang \\ Department of College English, Zhejiang Yuexiu University, Shaoxing, China \\ Email:964299532@qq.com
}

How to cite this paper: Huang, Y. (2021) A Narrative Study on Postmodern War Fiction Si je t'oublie, Bagdad. Open Access Library Journal, 8: e7632.

https://doi.org/10.4236/oalib.1107632

Received: June 10, 2021

Accepted: September 21, 2021

Published: September 24, 2021

Copyright (C) 2021 by author(s) and Open Access Library Inc.

This work is licensed under the Creative Commons Attribution International License (CC BY 4.0).

http://creativecommons.org/licenses/by/4.0/

\section{(c) (i) Open Access}

\begin{abstract}
Inam Cachatchi's novel Si je t'oublie, Bagdad expressed the individual's existential dilemma between nations and races by replacing the traditional grand narrative with petit narration. Different from the Western discourse dominated Iraq war literature, the novel criticized the true nature of neo-war on violence, which entails the realization through the marginal woman's perspective, non-liner narration and poetic narrative language. This thesis intends to explore the psychological shock and mental trauma by focusing on the narrative strategies, especially the narrative point of view, female narrative voice, and its unique narrative mode in this novel.
\end{abstract}

\section{Subject Areas}

Culture, Literature

\section{Keywords}

Neo-War, Female Narrative Voice, Marginal Man, Petit Narrative

\section{1. 引言}

自 2003 年美国对伊拉克发动战争以来，伊拉克内乱肆虐、经济衰退，人 民一直生活在水深火热里。2003 年夏, 伊拉克裔女作家伊娜姆・卡恰齐(Inaam Kachachi, 1952-) 以 “伊拉克战争和 $9 \cdot 11$ ” 事件为时代背景, 创作了小说《如 果我忘记你, 巴格达》 (Si je t'oublie, Bagdad), 获得了 2009 年度阿拉伯语文 学最高奖项——“The International Prize for Arabic Fiction”。不同于以美国作 家为代表的西方主流话语主导下的伊拉克文学，伊娜姆・卡恰齐从受害方视 角切入, 以女主人公泽娜一家人的曲折经历为缩影，展现了被迫举家移民美 国 15 年后, 具有双重国籍的泽娜以阿拉伯语 - 英语译者的身份重归故国, 直 面处于战争焦土中的家乡和亲人的心理历程。小说通过描述战争亲历者的故 
事, 揭露了战争焦土中的伊拉克社会的无序和混乱, 表达了作者对被战争撕 碎的故国和人民的关切。

目前, 国内外对该小说的研究尚且不多, 国内仅有崔月明对小说中的身 份认同做出研究, 该篇硕士论文通过分析小说主人公一家在战争中的不幸际 遇, 从身份构建失败的原因出发, 着重分析移民群体的身份认同困境, 并针 对不同的女性, 深入研究不同文化背景下成长的女性, 探讨她们的自我身份 认知的危机。[1]国外学界则多见对该小说的书评, 如法国《玛丽安娜》杂志 称之为 “新时期最佳小说之一” ，因为这部石破天惊的作品在小说里描述了 一场双重悲剧: 美国的失败和伊拉克的耻辱; 法国《世界报》评论小说用精 准且饱含深情语言，描述了 “一个国家至深的痛楚和一个永远卡在两个国家 之间的女人。”[1]而埃及当代著名小说家艾哈迈德・沙卡尔也高度赞赏小说 简洁有力的语言表达，[1]观点鲜明地展现了阿拉伯国家的一系列问题。相关 研究多关注小说中的女性主义和阿拉伯民族身份认同问题，较少分析小说本 身的叙事特色和语言风格。基于此, 本文着重研究小说在后现代战争语境背 景下的叙事特点。从边缘人物的叙事声音、独特的小叙事模式出发，分析泽 娜面临的亲情、爱情、同胞情和工作职责间的矛盾冲突和困境, 探讨后现代 战争对人们带来的心理冲击和精神创伤。

\section{2. 后现代战争中的女性声音}

意大利作家安贝托 - 艾柯(Umberto Eco)将后现代战争界定为 “敌人身份 不确定的非正面的现代战争。” [2]在后现代战争中，传统战争里明确的战争 范围、武器和军人等依托甚至连敌人和赢家都已不复存在。随着经济全球化 和信息技术的不断发展，后现代战争中战争发起方的意图不再具有决定意义。 除正面战场对抗外，非正面战场的燍杀更为突出。由于涉及多方势力不断抗 衡, 尤其是跨国资本的介入, 最终使得各势力分布具有极大的不确定性和不 可预知性，从而导致战争双输的结局。2003 年，美国声称伊拉克有大规模杀 伤性武器, 要推翻萨达姆恐怖政权, 解放伊拉克人民。美国的敌方只是针对 萨达姆及其部下，并非所有伊拉克人民。然而，2020 年，美国总统特朗普也 曾公开承认伊拉克并没有所谓的大规模杀伤性武器, 萨达姆的恐怖独裁也不 复成立。因此，相对于传统战争而言，所谓的敌方身份事实上并不明确。时 至今日，伊拉克战争也并没有给伊拉克人民带来所谓的民主和公正，伊拉克 人民仍然生活在水深火热中, 这场战争没有赢家。

此外，伊拉克战争的后现代性还体现在 “超视距” 中。顾名思义，“超 视距” 即公众肉眼 “看不到的” 战争，这也是最典型的后现代战争的特征之 一。由于美国民众看不到美国摄影记者在战场的血腥暴力场面，尽管战争给 伊拉克人民带来的痛苦真实存在，但却因没被记录下来，受害方无法通过画 面向世界诉说自己的痛苦，所以战争给受害国人民带来的痛苦被推远到看不 见, 成了单方面的痛苦。人们的视角受制于媒体, 而媒体受制于军方。因此, 如果说后现代战争是痛苦最小化的战争, 那只是对美国而言。美方操纵了对 战争的影像记录，将一场本来血腥的战争虚拟成一场没有痛苦的游戏。小说 中描述泽娜应聘时重复的套话就是福克斯新闻锻造出的： “身负事关国家利 
益的使命, 我是一名战士, 渴望帮助它的政府、它的同胞、它的军队, 我们 美国的军队, 去那里推翻萨达姆, 解放尝尽苦澀的人民。” [3] (P10)根据 2003 年美国民意调查, $70 \%$ 的美国人认为, 萨达姆与 “ $9 \cdot 11$ ”袭击有关。小说中也 描述了“9・11”事件后, 美国在伊拉克战场急缺阿拉伯语译员的处境, 迫于生 计的泽娜受丰厚报酬 “一年九万七千美元一一包住包吃包漂白” 的吸引, 决 定参军好赚钱送弟弟去戒毒中心, 甚至进大学, 给母亲洗洗肺, 将幸福带给 家人, 将一切恢复如初。当时的美国正处在紧张的战备状态, 随处都能听见/ 看见出现在报纸头条, 国会议员演说中的浓浓战意, 由此可见伊拉克战争的 典型后现代性。实际上, 美国中情局检查了过去 20 年的 2 万份文件, 没有找 到萨达姆和基地组织的任何联系。这个数据背后, 都是美国媒体的 “功劳” 。 美国民众看到的, 都是一些片面的、被精心安排过的画面。“电视不断玩弄 着我们的感情。屏幕激发着我们的肾上腺素, 转播着大炮的轰鸣声、炮弹的 爆炸声、奔跑逃难者的骚乱声, 还有大火的影像, 烟雾的影像及受威胁的年 轻人惨白的面容, 这一切倒勾勒出了摄影师所意图表现的胜利迹象。” (p. 16) 就这样, 伊拉克战争赶走了萨达姆。但为此却赔上了整个伊拉克。自此, “巴 格达属于所有人，伊拉克也再没有了主人。”(p. 16)

伊拉克地处中亚幼发拉底河和底格里斯河流域, 自公元前 4000 年苏美尔 文明起至今日, 深受亚述文明、波斯文明、希腊文明尤其是伊斯兰文明的影 响。泽娜自小深受伊拉克文学, 尤其是古典诗歌的熏陶, 小说从泽娜的第一 人称叙述视角, 以诗意的语言、细淢的心理描写刻画了泽娜及其家人处于战 争泥沼中的遭遇, 营造出如歌般充满诗意的氛围。虽是以战争为背景但却不 见暴力血腥, 全书始终在朦胧中寄托着淡淡的哀愁。开篇独白道: “这壮丽 的悲伤让我相信, 我不再是一个普通的美国人, 而是一个生根他出的女人, 我的根在这历史的深处。就像有人手里紧握着一块木炭, 它燃烧着与众不同 的生命”。(p. 3)结尾也写道 “这里女人们的哭泣, 不是一项简单的爱好, 而 是一种生活方式, 一项经常操练的全民运动, 有时单练, 有时合练, 以此保 持他们的精神面貌。哭泣强健了她们的心肌, 降低了动脉血压; 哭泣带来的 精神快感有时甚至超过了酒醉。” (p. 177)紧接着, 作者又跨越了时空的藩篱, 通过非线性叙事的手法, 通过泽娜第一人称的口述回顾了自己的成长过程, 概述了童年在巴格达和外公外婆一起度过的美好时光, “他们幸福地看着她 长大, 看着她绕着自己转圈, 她用天使般的栗色小脸蛋满足着他们的期许。” (p. 69)作者采用回顾性叙事建构了外婆拉玛, 母亲芭图尔以及其他一系列生 动的伊拉克普通女性形象。在小说的第六章、第十七章和第二十章, “我” 和 “该死的小说家” 为争夺书写权在激烈交锋着: “我不愿让给那个该死的 小说家, 她来推开我想坐在电脑前, 和我肩并肩, 彷佛在表演双重奏, 不可 避免地要分享同一台钢琴! 她想和我一起敲击键盘, 来一首四手联弹, 或是 二十指连弹; 想和我一起书写这个回到巴格达祖屋的美国小女孩的故事。而 我, 我不要这个小说家坐在我身边, 我把她推地越远越好, 不让她肆意妄为。 她一转过身去, 我就又回到她身后, 一键删除她刚在屏幕上写下的字句。” （p. 26） “我” 渴求书写属于自己的故事, 而不是任由 “笨蛋小说家” 任意按 照二元对立模式将 “我” 书写成为一个单纯的职业产品, 一个无法无天的Y 
头。最终, 泽娜和女小说家的博亦以泽娜将小说家推进坑里消灭而告终, 她 最终得以在屏幕前写完属于自己的故事。通过后现代碎片化和拼接的手法, 二人内心独白和对话呈现出叙述的不可靠性, 并超越了传统战争小说中男性 规范的界限。

除此之外, 小说还描述了泽娜和乳母的儿子穆海门的爱情故事。因幼时 被迫逃难, 美国收留并给予了泽娜一家公民身份, 还提供了报酬丰厚的军队 译员工作, 她对美国是心存感激的。然而, 穆海门身为伊拉克军人, 经历过 两伊战争, 被俘入狱长达四年之久, 战争带给他的是对美国人无尽的憎恨。 因此, 二人注定无法跨越亲情和国情的鸿沟, 这是一份没有结果的爱, 是 “另 一种形式的死亡, 一段失败的生命。” (p. 179)尽管泽娜心怀远大抱负和理想, 自以为从事着崇高正义的事业, 不仅能效忠美国, 还能帮助同胞推翻暴君, 获得解放、民主和自由。带着对故乡至深的热爱, 泽娜在参与严酷的审讯同 胞时, 虽然能力有限, 但毫不掩饰对伊拉克人民的热爱, 仍试图帮助同胞解 决困难。然而, 残酷的现实证明, 凭她一己之力, 根本无法消弭两国人民间 深深的仇恨和裂痕, 结果她只能黯然离开。在这场战争中, 泽娜收获的是无 尽的痛苦和忧愁, “与精炼的蜂蜜一样的忧伤一一浓厚、粘稠、透亮”。(p. 179) 全书以女性视角描述战争历史, 实现了女性叙事权威的建构, 将伊拉克战前 战后的社会状态真实、具象地呈现在读者面前。

\section{3. “边缘人”的小叙事模式}

在传统的战争小说中, 英雄/史诗叙事是最典型的叙事结构。随着后现代 战争对宏大历史的消解, 小说等文学创作中的叙事形态也逐渐转型, 更多的 作家开始关注大历史下小人物的遭际和命运。这种独特的叙事风格即小叙事 (petit narrative)。法国哲学家 Jeancois Lyotard 最先提出小叙事的概念, 将其 和宏大叙事(grand narrative/metanarrative)对比, 认为小叙事拒绝宏大叙事常 见的单一的真理主题, 更关切宏大叙事中常被忽略的小事件, 重点展现被边 缘化的人物及其个人情感体验。[4] 小说《如果我忘记你, 巴格达》不再关注 战争中的某一场惨烈的战斗、或是具体的战役, 而是聚焦后现代战争背景下 小人物的经历, 更具体地呈现后现代战争对生命个体的巨大伤害。小说通篇 鲜有对残酷战争前线的直接暴力描写, 但作者却以边缘女性的视角和真情实 感的书写, 借助伊拉克人民战争前后生活图景的对比, 试图言说后现代战争 的暴力本质。

德国心理学家勒温 Park - Robert Ezra 在“Human migration and the marginal man”中将边缘人 (marginal man) 界定为受社会动荡后失去身份但无法与 时俱进的一群人。[5]他们游离在两个社会群体之间, 都不能完全参与其中。 由于无法建构新的身份, 不能适应新的环境, 被迫脱离主流社会, 被排除在 核心群体之外。泽娜就具有边缘人的典型特质, 在两个国家都不被接纳, 从 而内心饱受煎謷。她本是个伊拉克摩苏尔的平凡姑娘, 父亲萨巴赫 - 巴赫纳 姆是一名优雅的电视台主持人, 母亲芭图尔是大学老师, 一家人对泽娜的出 现倍感幸福, 有了她家里 “充满了爱抚与欢笑。” (p. 69)一家人原本在伊拉 克平凡且愉快地生活着。然而, 这位就连自己影子都害怕的优雅主持人却被 
阴谋化身为反对党和革命, 被捕入狱遭遇了一系列的酷刑, 被迫脱光衣服毒 打 “像只蛆虫”, 甚至被判处绞刑。被家人救回来后, 因一截舌头被钳子夹 下, 他不能说话, 牙齿被打碎, 满身都是惣伤, 眼泪也不断地流, “彷佛眼 皮底下装了蓄水池。” (p. 70)讽刺的是, 这一系列遭遇却是源于他最亲近的 同事举报, “他批评电视新闻的长度, 不满新闻像是炒冷饭。” (p. 70)由于 时局无序混乱, 迫于形势, 控诉无门的母亲再也不愿留下这个国家, 她决定 抛下一切, 一家人隐姓埋名, 举家逃难到美国, 只留下年迈的外婆拉玛独自 留在巴格达。“离开即是背叛, 背叛了国家, 背叛了灌溉这国家的底格里斯 河和幼发拉底河, ” (p. 9)当一家人历经艰辛从难民蜕变为美利坚公民后, 母 亲却发出来痛苦的咕噜声, “平常纯白的面孔变得通红通红, 像是发烧的病 人, 眼泪大颗大颗从她的眼中淌下, 随后在她采热的脸项上蒸发不见, ”(p. 21) 小弟弟雅善也在美国染上毒瘾, 父亲也终日郁郁寡欢, 一家人的生活似乎并 没有因为拥有 “稀罕的” 美国国籍而好转, “我们变成了神经脆弱的生物, 发 出愤怒却又恐惧的嘶吼。我们用手抵住额头或是捂住嘴巴; “噢, 天啊! …… 噢, 天啊! , 不断重复着这句话, 彷佛我们忘却了自己的语言, 仿佛我们的 所有词汇里只剩下这三个字。”(p. 11)

为了新祖国的利益, 十五年后, 泽娜转而重归故国当翻译, “所有的回 归都受到欢迎, 除了这次回归; 所有的怀抱都张开迎接每一个回头的浪子, 除了这个姑娘。” (p. 72) 虽然泽娜并未直接参战, 但她被迫夹在两种截然不 同的文化间, 在政治和历史的夹缝当中, 在意志与命运的纠葛中委屈无奈近 乎于怒: “我是一只有两个窝的狗”, “我质疑自己的身份: “是伪君子、两 面派的美国人? 还是暂时沉睡中的伊拉克人? 就和今年我们派向敌区的那些 沉睡中的间谍一样? 那么我为什么对受害者感到怜悯呢? ” ( p. 178)泽娜无 奈地目睹着美军炮弹在巴格达肆虐, 在美国的袭击下, 巴格达不得不屈服。 在泽娜看来, 两国交战就好比 “用母亲的打火机点燃自己的头发, 或使用剪 刀撕破自己的皮肤, 甚或是用自己的右手给左脸来了一记响亮的耳光。” ( 178)当她斗志昂扬地回到伊拉克, 试图对同胞微笑时, 也没有得到想象中的 热烈欢迎。饱受战争冲击的同胞对她报以恐惧和憎恨的眼神: “他们应该张 开双臂欢迎这些从车上新来的人, 还是应该朝他们脸上吐唾沫? ” (p. 61)

不仅如此, 外婆拉玛 - 法图伊也重归祖国的姑娘充满质疑, 为美国人工 作就是与侵略者为伍, 她的这次回归伤透了她们的肺腑, 因此需要对这个无 知的没有教养的女孩进行再教育。她用双手轻轻拍着大腿, 做着这通常在丧 礼或是遇到大灾难时做的动作。不难看出, 外婆对泽娜译员身份感到差耻愤 怒又绝望。在她忧伤的眼神里, “我已经看出了整部电影的情节。脆弱又顺 从的我等待着即将到来的说教, 我明白自己的过错, 也完全不想为自己辩护。” (p. 77)然而, 外婆只是不知疲倦地重复着外公尤瑟夫 - 萨乌尔上校昔年的故 事。在被迫因民族主义退伍后, 他满腔的爱国心都倾注在黄褐色的军装上, 每年一月六日的建军节他都会认真擦拭制服上的星星, 渴望政权改组时能被 通知重新加入军队。军装成为外婆教育泽娜的最佳教具, “她用颤抖的手帮 我穿上这件镶着金色星星的衣服……这个疯狂的时代, 一件伊拉克上校的军 装怎么能养出一件美国产的防弹衣? ” (p. 83)这和美国军队提供的完全不同, 
美式军装没有用这般完美讲究的剪裁, 虽是最新式的布料, 但却只是最实际 的做工。然而这样的迷彩服却能让泽娜内心充满骄傲, 希冀身着迷彩服, 偿 还美国从小接纳她们一家的恩情。同时还能解放伊拉克，帮助同胞们获得自 由, “老人们将浸润青春之泉, 呷饮民主的乳汁, 每个人都能品尝生命的味 道, 一如我在这里体味到的一样。” (p. 10)然而, 她百般努力之后, 泽娜仍 身着占领军的黄褐色戎装, 甚至最终被 “我” 的工作和军装气死。

通过泽娜对外婆拉玛，外公尤瑟夫、母亲芭图尔、父亲巴赫内姆等回忆 的插叙，小说展现了泽娜身处亲情、同胞情和工作职责的巨大矛盾和冲突的 图圄。诸多性格鲜明的人物形象，为读者生动再现了伊拉克广大民众受到的 战争冲击。

\section{4. 结语}

战争叙事离不开所处的种族、环境及其时代，作为历史记忆的一部分， 是对时代社会特征的反映。作为典型的后现代战争，伊拉克战争由于给当地 人民带来了深重持久的苦难, 因而具有难以言说性。直到十多年后, 才逐渐 涌现出以伊拉克战争为背景的文学作品。其中, 大多都是以英美作家为代表 的西方主流视角为主导。伊娜姆 - 卡恰齐颠覆了传统战争小说的宏大叙事, 以典型的后现代碎片拼贴和小叙事模式，为战争中的边缘女性发声，探寻了 后现代战争对人们造成的心理冲击和精神创伤。

本文以后现代战争语境下的叙事技巧为切入点，一定程度地反思了后现 代战争的暴力本质，从而批判伊拉克战争带给国家、民族和人民的深重苦难。 该小说内容十分丰富，涉及民族、宗教、军事、政治等多元主题，小说在诸 多细节描述和情节安排中埋下诸多伏笔，如泽娜在巴格达、提克里特等地亲 眼目睹了美军对伊拉克普通民众的暴力行径，曾经气势恢宏的伊拉克传统建 筑在战争中饱受摧残的细淢描写等, 无不向读者生动展现了战争对伊拉克传 统文化带来的毁灭性破坏。然篇幅有限, 本文仅从叙事角度对小说做出分析, 其他方面的内容和表现形式仍值得进一步深入研究和探讨。

\section{Conflicts of Interest}

The author declares no conflicts of interest.

\section{References}

[1] 崔月明. 小说《美国孙女》中的身份认同研究[D]: [硕士学位论文]. 黑龙江: 黑龙 江大学, 2018.

[2] Eco, U. (2007) Trans. Alastair McEwen, Turning Back the Clock: Hot Wars and Media Populism. Houghton Mifflin Harcourt, Boston.

[3] 伊娜姆 - 卡恰齐. 如果我忘记你，巴格达 [M]. 林盛译. 北京: 人民文学出版社， 2012.

[4] Readings, B. (1991) Introducing Lyotard Art and Politics. Taylor \& Francis, London, 47-51.

[5] Park, R.E. (1928) Human Migration and the Marginal Man. The American Journal of Sociology, 33, 881-893. https://doi.org/10.1086/214592 


\section{Appendix (Abstract and Keywords in Chinese)}

\section{后现代战争语境下《如果我忘记你, 巴格达》的叙事研究}

摘要：伊拉克裔女作家伊娜姆 - 卡恰齐的小说《如果我忘记你, 巴格达》摒 弃了传统战争小说的宏大叙事模式, 以边缘女性的亲历视角、时空穿插的非 线性叙事手法，具象化再现了挣扎在国家和民族两难中无法选择的个体生存 困境。不同于以西方主流话语为主导的伊拉克战争文学，伊娜姆・卡恰齐用 诗性的叙事风格, 试图言说被入侵国人民的心理创伤，从而深刻批判后现代 战争的暴力本质。

关键词：后现代战争，女性叙事声音，边缘人，小叙事 